J. Nonlinear Var. Anal. 2 (2018), No. 2, pp. 165-175

Available online at http://jnva.biemdas.com

https://doi.org/10.23952/jnva.2.2018.2.05

\title{
EDELSTEIN'S FIXED POINT THEOREM IN SEMIMETRIC SPACES
}

\author{
TOMONARI SUZUKI
}

Department of Basic Sciences, Faculty of Engineering, Kyushu Institute of Technology, Tobata, Kitakyushu 804-8550, Japan

\begin{abstract}
Very recently, Kirk and Shahzad gave one open question on the Edelstein's fixed point theorem in compact metric spaces proved in 1962. In this paper, we give a negative answer to this question. We extend the Edelstein's theorem to semimetric spaces. We also study contractive conditions.
\end{abstract}

Keywords. Contraction; Edelstein's fixed point theorem; Semimetric space.

2010 Mathematics Subject Classification. 54H25, 54E25.

\section{INTRODUCTION}

In 1962, Edelstein proved the following famous fixed point theorem.

Theorem 1.1 (Edelstein [5]). Let $(X, d)$ be a compact metric space and let $T$ be a mapping on $X$ satisfying

$$
x \neq y \quad \Rightarrow \quad d(T x, T y)<d(x, y)
$$

for any $x, y \in X$. Then $T$ has a unique fixed point $z$. Moreover, $\left\{T^{n} x\right\}$ converges to zfor all $x \in X$.

Several generalizations of Theorem 1.1 have been proved; see, for example, [11, 16, 18, 20, 23, 25]. Very recently, Kirk and Shahzad in [14] extended Theorem 1.1 to strong quasimetric spaces; see Theorem 5.1. They also gave one open question (Question 5.1).

In this paper, we give a negative answer to Question 5.1 (see Example 5.1). Motivated by this fact, we extend Theorem 1.1 to semimetric spaces (see Theorem 4.2). We also study contractive conditions (see Section 3).

\section{PRELIMINARIES}

Throughout this paper, we denote by $\mathbb{N}, \mathbb{Z}, \mathbb{Q}$ and $\mathbb{R}$ the sets of all positive integers, all integers, all rational numbers and all real numbers, respectively. For a real number $t$, we denote by $[t]$ the maximum integer not exceeding $t$.

In this section, we give some preliminaries.

Definition 2.1. Let $X$ be a nonempty set and let $d$ be a function from $X \times X$ into $[0, \infty)$. Then $(X, d)$ is said to be a semimetric space if the following hold:

(D1) $d(x, x)=0$.

E-mail address: suzuki-t@mns.kyutech.ac.jp.

Received December 31, 2017; Accepted February 12, 2018.

(C)2018 Journal of Nonlinear and Variational Analysis 
(D2) $d(x, y)=0 \Rightarrow x=y$.

(D3) $d(x, y)=d(y, x)$.

Definition 2.2. Let $(X, d)$ be a semimetric space. Let $\left\{x_{n}\right\}$ be a sequence in $X$ and let $x \in X$. Let $\kappa \in \mathbb{N}$ and let $T$ be a mapping on $X$.

- $\left\{x_{n}\right\}$ is said to converge to $x$ if $\lim _{n} d\left(x_{n}, x\right)=0$.

- $X$ is said to be sequentially compact if every sequence in $X$ has a convergent subsequence.

- $X$ is said to be Hausdorff if $\lim _{n} d\left(x_{n}, x\right)=0$ and $\lim _{n} d\left(x_{n}, y\right)=0$ imply $x=y$.

- $X$ is said to be $\kappa$-Hausdorff if

$$
\lim _{n \rightarrow \infty} D\left(x, u_{n}^{(1)}, \cdots, u^{(\kappa)}, y\right)=0
$$

implies $x=y$, where

$$
\begin{aligned}
& D\left(x, u^{(1)}{ }_{n}, \cdots, u^{(\kappa)}{ }_{n}, y\right) \\
& =d\left(x, u^{(1)}{ }_{n}\right)+d\left(u^{(1)}{ }_{n}, u^{(2)}{ }_{n}\right)+\cdots+d\left(u^{(\kappa-1)}{ }_{n}, u^{(\kappa)}{ }_{n}\right)+d\left(u^{(\kappa)}{ }_{n}, y\right) .
\end{aligned}
$$

- $d$ is said to be sequentially continuous if $\lim _{n} d\left(x_{n}, y_{n}\right)=d(x, y)$ provided $\left\{x_{n}\right\}$ and $\left\{y_{n}\right\}$ converge to $x$ and $y$, respectively.

- $T$ is said to be sequentially continuous if $\left\{T x_{n}\right\}$ converges to Tx provided $\left\{x_{n}\right\}$ converges to $x$.

\section{Remark 2.1.}

- It is obvious that $X$ is Hausdorff $\Leftrightarrow X$ is 1-Hausdorff.

- It is also obvious that $X$ is $\lambda$-Hausdorff $\Rightarrow X$ is $\kappa$-Hausdorff provided $\kappa<\lambda$.

The following is essentially proved in [22]. For the sake of completeness, we give a proof.

Proposition 2.1 ([22]). Let $(X, d)$ be a semimetric space. Assume that d is sequentially continuous. Then $X$ is 2-Hausdorff.

Proof. Suppose $\lim _{n} D\left(x, u_{n}, v_{n}, y\right)=0$. Then $\left\{u_{n}\right\}$ converges to $x$ and $\left\{v_{n}\right\}$ converges to $v$. So we have $d(x, y)=\lim _{n} d\left(u_{n}, v_{n}\right)=0$. Thus, we obtain $x=y$.

\section{Contractive Conditions}

In this section, we study contractive conditions.

Definition 3.1. Let $X$ be a nonempty set and let $d$ be a function from $X \times X$ into $[0, \infty)$. Let $T$ be a mapping on $X$.

(1) $T$ is said to be an Edelstein contraction [5] if $d(T x, T y)<d(x, y)$ for any $x, y \in X$ with $d(T x, T y)>$ 0 .

(2) $T$ is said to be a CJM contraction $[4,8,15]$ if the following hold:

(2-i) For any $\varepsilon>0$, there exists $\delta>0$ such that $d(x, y)<\varepsilon+\delta$ implies $d(T x, T y) \leq \varepsilon$.

(2-ii) $T$ is Edelstein.

(3) $T$ is said to be a Browder contraction [2] if there exists a function $\varphi$ from $[0, \infty)$ into itself satisfying the following:

(3-i) $\varphi$ is nondecreasing and right continuous.

(3-ii) $\varphi(t)<t$ for any $t \in(0, \infty)$. 
(3-iii) $d(T x, T y) \leq \varphi \circ d(x, y)$ for all $x, y \in X$.

(4) $T$ is said to be a contraction [1,3] if there exists $r \in[0,1)$ satisfying $d(T x, T y) \leq r d(x, y)$ for all $x, y \in X$.

Remark 3.1. The definition on Edelstein contraction in Definition 3.1 (1) differs from the assumption in Theorem 1.1. However, the following proposition ensure that both are equivalent under (D1) and (D2).

Proposition 3.1. Let $X$ be a nonempty set and let $d$ be a function from $X \times X$ into $[0, \infty)$. Assume (D1) and (D2) in Definition 2.1. Let $T$ be an Edelstein contraction on $X$. Then the following are equivalent:

(i) $T$ is Edelstein.

(ii) $d(T x, T y)<d(x, y)$ for any $x, y \in X$ with $d(x, y)>0$.

Proof. We first show (i) $\Rightarrow$ (ii). We assume (i). Let $x, y \in X$ satisfy $d(x, y)>0$. We consider the following two cases:

- $d(T x, T y)=0$.

- $d(T x, T y)>0$.

In the first case, we have $d(T x, T y)=0<d(x, y)$. In the second case, from (i), we have $d(T x, T y)<$ $d(x, y)$. Thus, (ii) holds.

Conversely we assume (ii). Let $x, y \in X$ satisfy $d(T x, T y)>0$. From (D1), we have $T x \neq T y$. So we obtain $x \neq y$. From (D2), we have $d(x, y)>0$. So, from (ii), we have $d(T x, T y)<d(x, y)$. Thus, (i) holds.

In order to study the Browder and Boyd-Wong contractive conditions, Hegedüs and Szilágyi in [7] considered subsets of $[0, \infty)^{2}$. We give definitions which are strongly connected with contractive conditions in Definition 3.1.

Definition 3.2. Let $Q$ be a subset of $[0, \infty)^{2}$.

(1) $Q$ is said to be Edelstein if $u>0$ implies $u<t$ for any $(t, u) \in Q$.

(2) $Q$ is said to be $C J M$ if the following hold:

(2-i) For any $\varepsilon>0$, there exists $\delta>0$ such that $u \leq \varepsilon$ holds for any $(t, u) \in Q$ with $t<\varepsilon+\delta$.

(2-ii) $Q$ is Edelstein.

(3) $Q$ is said to be a Browder if there exists a function $\varphi$ from $[0, \infty)$ into itself satisfying the following:

(3-i) $\varphi$ is nondecreasing and right continuous.

(3-ii) $\varphi(t)<t$ for any $t \in(0, \infty)$.

(3-iii) $u \leq \varphi(t)$ for any $(t, u) \in Q$.

(4) $Q$ is said to be contractive if there exists $r \in[0,1)$ such that $u \leq r t$ holds for any $(t, u) \in Q$.

The following obviously holds. See also Proposition 6 in [17].

Proposition 3.2. Let $X$ be a nonempty set and let $d$ be a function from $X \times X$ into $[0, \infty)$. Let $T$ be a mapping on $X$. Define a subset $Q$ of $[0, \infty)^{2}$ by

$$
Q=\{(d(x, y), d(T x, T y)): x, y \in X\} .
$$

Then the following hold: 
(i) $T$ is an Edelstein contraction iff $Q$ is Edelstein.

(ii) $T$ is a CJM contraction iff $Q$ is CJM.

(iii) $T$ is a Browder contraction iff $Q$ is Browder.

(iv) $T$ is a contraction iff $Q$ is contractive.

Definition 3.3 ([19]). Let $Q$ be a subset of $[0, \infty)^{2}$.

(1) $Q$ is said to satisfy Condition $C(0,0,0)$ if the following hold:

(1-i) $u<t$ for any $(t, u) \in Q$ with $u>0$.

(1-ii) There does not exist $\tau>0$ and a sequence $\left\{\left(t_{n}, u_{n}\right)\right\}$ in $Q$ satisfying $\tau<t_{n}, \tau<u_{n}$ and $\lim _{n} t_{n}=\lim _{n} u_{n}=\tau$.

(2) $Q$ is said to satisfy Condition $C(0,0,1)$ if the following hold:

(2-i) $Q$ satisfies Condition $\mathrm{C}(0,0,0)$.

(2-ii) There does not exist $\tau>0$ and a sequence $\left\{\left(t_{n}, u_{n}\right)\right\}$ in $Q$ satisfying $\tau<t_{n}, u_{n}=\tau$ and $\lim _{n} t_{n}=\tau$.

(3) $Q$ is said to satisfy Condition $C(0,0,2)$ if the following hold:

(3-i) $Q$ satisfies Condition $\mathrm{C}(0,0,0)$.

(3-ii) There does not exist $\tau>0$ and a sequence $\left\{\left(t_{n}, u_{n}\right)\right\}$ in $Q$ satisfying $\tau<t_{n}, u_{n} \leq \tau$ and $\lim _{n} t_{n}=\lim _{n} u_{n}=\tau$.

(4) $Q$ is said to satisfy Condition $C(0,1,0)$ if the following hold:

(4-i) $Q$ satisfies Condition $\mathrm{C}(0,0,0)$.

(4-ii) There does not exist $\tau>0$ and a sequence $\left\{\left(t_{n}, u_{n}\right)\right\}$ in $Q$ satisfying $t_{n}=\tau, u_{n}<\tau$ and $\lim _{n} u_{n}=\tau$.

(5) $Q$ is said to satisfy Condition $C(1,0,0)$ if the following hold:

(5-i) $Q$ satisfies Condition $\mathrm{C}(0,0,0)$.

(5-ii) There does not exist $\tau>0$ and a sequence $\left\{\left(t_{n}, u_{n}\right)\right\}$ in $Q$ satisfying $t_{n}<\tau, u_{n}<\tau$ and $\lim _{n} t_{n}=\lim _{n} u_{n}=\tau$.

(6) Let $(p, q, r) \in\{0,1\}^{2} \times\{0,1,2\}$. Then $Q$ is said to satisfy Condition $C(p, q, r)$ if $Q$ satisfies Conditions $\mathrm{C}(p, 0,0), \mathrm{C}(0, q, 0)$ and $\mathrm{C}(0,0, r)$.

Remark 3.2. The expressions on the above conditions are a little different from those in [19]. Of course, both are essentially the same.

The following is essentially proved in [19].

Proposition 3.3 ([19]). Let $Q$ be a subset of $[0, \infty)^{2}$. Then the following hold:

(i) $Q$ is CJM iff $Q$ satisfies Condition $C(0,0,0)$.

(ii) $Q$ is Browder iff $Q$ satisfies Condition $C(1,1,2)$.

Proposition 3.4. Let $Q$ be a closed subset of $[0, \infty)^{2}$. Then the following are equivalent:

(i) $Q$ is Edelstein.

(ii) $Q$ is $C J M$.

(iii) $Q$ is Browder.

Proof. (iii) $\Rightarrow$ (ii) and (ii) $\Rightarrow$ (i) are obvious. 
Let us prove (i) $\Rightarrow$ (iii). We assume (i). Arguing by contradiction, we assume that $Q$ does not satisfy Condition $\mathrm{C}(1,1,2)$. Then there exist $\tau>0$ and a sequence $\left\{\left(t_{n}, u_{n}\right)\right\}$ in $Q$ satisfying $\lim _{n} t_{n}=\lim _{n} u_{n}=\tau$. Since $Q$ is closed, we have $(\tau, \tau) \in Q$. This is a contradiction. Therefore we have shown that $Q$ satisfies Condition $\mathrm{C}(1,1,2)$. By Proposition 3.3 (ii), we obtain that $Q$ is Browder.

\section{FiXed Point Theorems}

The purpose of this section is to prove the following two fixed point theorems:

Theorem 4.1. Let $(X, d)$ be a sequentially compact, 2-Hausdorff semimetric space. Let $T$ be a CJM contraction on $X$. Then $T$ has a unique fixed point $z$. Moreover, $\left\{T^{n} x\right\}$ converges to $z$ for all $x \in X$.

Theorem 4.2. Let $(X, d)$ be a sequentially compact semimetric space. Assume that $d$ is sequentially continuous. Let $T$ be an Edelstein contraction on $X$. Then $T$ has a unique fixed point $z$. Moreover, $\left\{T^{n} x\right\}$ converges to zfor all $x \in X$.

We begin with the following lemma.

Lemma 4.1. Let $\left\{a_{n}\right\}$ be a sequence in $[0, \infty)$. Then the conjunction of (a-1) and (a-2) is equivalent to the conjunction of (b-1), (b-2) and (b-3).

(a-1) For any $\varepsilon>0$, there exists $\delta>0$ such that $a_{n}<\varepsilon+\delta$ implies $a_{n+1} \leq \varepsilon$.

(a-2) $a_{n+1}>0$ implies $a_{n+1}<a_{n}$.

(b-1) $\left\{a_{n}\right\}$ is nonincreasing.

(b-2) $a_{n}>0$ implies $a_{n+1}<a_{n}$.

(b-3) $\left\{a_{n}\right\}$ converges to 0 .

Proof. We assume (a-1) and (a-2). In order to show (b-1), we fix $n \in \mathbb{N}$. We consider the following two cases:

$$
\text { - } a_{n+1}=0 \text {. } a_{n+1}>0 \text {. }
$$

In the first case, we have $a_{n+1}=0 \leq a_{n}$. In the second case, from (a-2), we have $a_{n+1}<a_{n}$. Thus, (b-1) holds. We can similarly prove (b-2). Let us prove (b-3). From (b-1), $\left\{a_{n}\right\}$ converges to some $\varepsilon \geq 0$. Arguing by contradiction, we assume $\varepsilon>0$. We choose $\delta>0$ appearing in (a-1). From the definition of $\varepsilon$, we can choose $v \in \mathbb{N}$ satisfying $a_{v}<\varepsilon+\delta$. Then we have $\varepsilon \leq a_{v+1} \leq \varepsilon$. Hence

$$
a_{v+1}=a_{v+2}=\varepsilon>0
$$

holds. From (a-2), we have

$$
\varepsilon=a_{v+2}<a_{v+1}=\varepsilon,
$$

which implies a contradiction. Therefore we obtain $\varepsilon=0$. Thus, (b-3) holds.

Next, we assume (b-1), (b-2) and (b-3). In order to prove (a-2), suppose $a_{n+1}>0$. From (b-1), we have $0<a_{n+1} \leq a_{n}$. From (b-2), we have $a_{n+1}<a_{n}$. Thus, (a-2) holds. Let us prove (a-1). Fix $\varepsilon>0$. We consider the following two cases:

- $a_{1} \leq \varepsilon$.

- $a_{v+1} \leq \varepsilon<a_{v}$ for some $v \in \mathbb{N}$. 
In the first case, we put $\delta=1$. We put $v=0$ and $a_{0}=\infty$ temporarily. In the second case, we put $\delta:=a_{v}-a_{v+1}>0$. We fix $n \in \mathbb{N}$ with $a_{n}<\varepsilon+\delta$. Then since $a_{n}<a_{v}$ holds, we have $n \geq v+1$. We have

$$
a_{n+1} \leq a_{v+2} \leq a_{v+1} \leq \varepsilon
$$

Therefore we have shown (a-1).

Now we give a proof of Theorem 4.1.

Proof of Theorem 4.1. We first show

$$
d(T x, T y) \leq d(x, y) \text { for all } x, y \in X .
$$

Indeed, since $T$ is CJM, we note that $T$ is Edelstein. We consider the following two cases:

- $d(T x, T y)=0$.

- $d(T x, T y)>0$.

In the first case, we have $d(T x, T y)=0 \leq d(x, y)$. In the second case, we have $d(T x, T y)<d(x, y)$. Thus, (4.1) holds.

We next show

$$
\lim _{n \rightarrow \infty} d\left(T^{n} x, T^{n+1} x\right)=0 \quad \text { for all } x \in X
$$

Indeed, since $T$ is CJM, the following hold:

(i) For any $\varepsilon>0$, there exists $\delta>0$ such that $d(x, T x)<\varepsilon+\delta$ implies $d\left(T x, T^{2} x\right) \leq \varepsilon$.

(ii) $d\left(T x, T^{2} x\right)>0$ implies $d\left(T x, T^{2} x\right)<d(x, T x)$.

So by Lemma 4.1, we obtain (4.2).

Fix $u \in X$. From (4.2), we have $\lim _{n} d\left(T^{n} u, T^{n+1} u\right)=0$. Since $X$ is sequentially compact, there exists a subsequence $\{f(n)\}$ of the sequence $\{n\}$ in $\mathbb{N}$ such that $\left\{T^{f(n)} u\right\}$ converges to some $z \in X$. We have from (4.1)

$$
\lim _{n \rightarrow \infty} d\left(T^{f(n)+1} u, T z\right) \leq \lim _{n \rightarrow \infty} d\left(T^{f(n)} u, z\right)=0 .
$$

It is obvious that $\lim _{n} d\left(T^{f(n)} u, T^{f(n)+1} u\right)=0$ holds. Thus,

$$
\lim _{n \rightarrow \infty} D\left(z, T^{f(n)} u, T^{f(n)+1} u, T z\right)=0
$$

holds. From 2-Hausdorffness of $X$, we obtain $T z=z$, that is, $z$ is a fixed point of $T$. From (4.1), we have

$$
d\left(T^{n+1} u, z\right)=d\left(T \circ T^{n} u, T z\right) \leq d\left(T^{n} u, z\right),
$$

thus, $\left\{d\left(T^{n} u, z\right)\right\}$ is nonincreasing. So, we obtain $\lim _{n} d\left(T^{n} u, z\right)=0$.

Arguing by contradiction, we suppose that $w$ is a fixed point of $T$ that is differ from $z$. Then we have $d(T z, T w)=d(z, w)>0$. So we obtain

$$
d(z, w)=d(T z, T w)<d(z, w),
$$

which implies a contradiction. Therefore the fixed point $z$ is unique.

Remark 4.1. From the above proof, we can weaken the assumption on $T$ as follows: $T$ is Edelstein and $T$ satisfies (i) in the proof.

By Theorem 4.1, we obtain the following corollary, which is a generalization of the Banach contraction principle [1,3]. See [10, 21, 22] for other generalizations. 
Corollary 4.1. Let $(X, d)$ be a sequentially compact, 2-Hausdorff semimetric space. Let $T$ be a contraction on $X$. Then $T$ has a unique fixed point $z$. Moreover, $\left\{T^{n} x\right\}$ converges to $z$ for all $x \in X$.

In order to generalize Theorem 1.1, we prove the following lemma:

Lemma 4.2. Let $(X, d)$ be a sequentially compact semimetric space. Assume that $d$ is sequentially continuous. Let $T$ be a sequentially continuous mapping on $X$. Define a subset $Q$ of $[0, \infty)^{2}$ by (3.1). Then $Q$ is compact.

Proof. Let $\left\{\left(t_{n}, u_{n}\right)\right\}$ be a sequence in $Q$. Then there exist sequences $\left\{x_{n}\right\}$ and $\left\{y_{n}\right\}$ in $X$ satisfying

$$
d\left(x_{n}, y_{n}\right)=t_{n} \quad \text { and } \quad d\left(T x_{n}, T y_{n}\right)=u_{n}
$$

for $n \in \mathbb{N}$. Since $X$ is sequentially compact, there exists a subsequence $\{f(n)\}$ of $\{n\}$ in $\mathbb{N}$ such that $\left\{x_{f(n)}\right\}$ and $\left\{y_{f(n)}\right\}$ converge to $x$ and $y$, respectively. Since $T$ is sequentially continuous, $\left\{T x_{f(n)}\right\}$ and $\left\{T y_{f(n)}\right\}$ converge to $T x$ and $T y$, respectively. Since $d$ is sequentially continuous, we obtain

$$
t:=d(x, y)=\lim _{n \rightarrow \infty} d\left(x_{f(n)}, y_{f(n)}\right)=\lim _{n \rightarrow \infty} t_{f(n)}
$$

and

$$
u:=d(T x, T y)=\lim _{n \rightarrow \infty} d\left(T x_{f(n)}, T y_{f(n)}\right)=\lim _{n \rightarrow \infty} u_{f(n)} .
$$

Thus, $\left\{\left(t_{f(n)}, u_{f(n)}\right)\right\}$ converges to $(t, u) \in Q$.

Proposition 4.1. Let $(X, d)$ be a sequentially compact semimetric space. Assume that $d$ is sequentially continuous. Let $T$ be a mapping on $X$. Then the following are equivalent:

(i) $T$ is Edelstein.

(ii) $T$ is $C J M$.

(iii) $T$ is Browder.

Proof. (iii) $\Rightarrow$ (ii) and (ii) $\Rightarrow$ (i) are obvious.

Let us prove (i) $\Rightarrow$ (iii). We assume (i). As in the proof of Theorem 4.1, we can prove (4.1). Let $\left\{x_{n}\right\}$ be a sequence in $X$ converging to some $x \in X$. From (4.1), we have

$$
\lim _{n \rightarrow \infty} d\left(T x_{n}, T x\right) \leq \lim _{n \rightarrow \infty} d\left(x_{n}, x\right)=0 .
$$

So, $T$ is sequentially continuous. Define a subset $Q$ of $[0, \infty)^{2}$ by (3.1). By Proposition 3.2 (i), $Q$ is Edelstein. By Lemma 4.2, $Q$ is compact. Hence $Q$ is closed. By Proposition 3.4, $Q$ is Browder. By Proposition 3.2 (iii), $T$ is Browder.

We give a proof of Theorem 4.2.

Proof of Theorem 4.2. By Proposition 2.1, $X$ is 2-Hausdorff. Also, by Proposition 4.1, $T$ is CJM. So by Theorem 4.1, we obtain the desired result. 


\section{KIRK-SHAHZAD's QUESTION}

We give a negative answer to Kirk-Shahzad's question raised in [14]. We first state the question.

Definition $5.1([12,13])$. Let $X$ be a nonempty set and let $d$ be a function from $X \times X$ into $[0, \infty)$. Then $(X, d)$ is said to be a strong quasimetric space or a strong b-metric space if (D1)-(D3) and the following hold:

(SQ3) There exists $K \geq 1$ satisfying $|d(x, z)-d(x, y)| \leq K d(y, z)$ for all $x, y, z \in X$.

Definition 5.2 ([6]). Let $X$ be a nonempty set and let $d$ be a function from $X \times X$ into $[0, \infty)$. Then $(X, d)$ is said to be a $K$-relaxed polygonal metric space or a p-metric space if (D1)-(D3) and the following hold:

(p3) There exists $K \geq 1$ satisfying

$$
d(x, y) \leq K D\left(x, u_{1}, u_{2}, \cdots, u_{n}, y\right)
$$

for all $n \in \mathbb{N}$ and $x, y, u_{1}, \cdots, u_{n} \in X$.

Theorem 5.1 (Theorem 4.6 in [14]). Let $(X, d)$ be a compact strong quasimetric space and let $T$ be a mapping on $X$ satisfying (1.1) for any $x, y \in X$. Then $T$ has a unique fixed point $z$. Moreover, $\left\{T^{n} x\right\}$ converges to $z$ for all $x \in X$.

Question 5.1 (page 2206 in [14]). Does Theorem 5.1 hold if $X$ is merely assumed to be a p-metric space or, more generally, a quasimetric space?

We give a negative answer to Question 5.1.

Lemma 5.1. Let $X$ be a nonempty subset of $\mathbb{R}$ and let $\theta \in \mathbb{R} \backslash \mathbb{Q}$. Define a relation $\sim$ on $X$ as follows: $x \sim y$ iff there exist $k, \ell \in \mathbb{Z}$ satisfying

$$
y-x=k \theta+\ell
$$

Then the following hold:

(i) $\sim$ is an equivalence relation.

(ii) $(k, \ell) \in \mathbb{Z}^{2}$ is uniquely determined.

Proof. We can easily prove (i). Also, noting that $\{1, \theta\}$ is linear independent over $\mathbb{Q}$, we can obtain (ii) easily.

Lemma 5.2 ([6]). Let $X$ be a nonempty set and let $d$ be a function from $X \times X$ into $[0, \infty)$ satisfying (D1)-(D3). Let $K \in[1, \infty)$. Then the following are equivalent:

- (p3) with $K$ holds.

- There exist a metric $\rho$ on $X$ satisfying

$$
\rho(x, y) \leq d(x, y) \leq K \rho(x, y) .
$$

for $x, y \in X$.

Lemma 5.3. Let $(X, \rho)$ be a metric space. Let $\alpha$ be a function from $X \times X$ into $[1, K]$, where $K \in[1, \infty)$ is some real number. Assume $\alpha(x, y)=\alpha(y, x)$ for $x, y \in X$. Define a function d from $X \times X$ into $[0, \infty)$ by

$$
d(x, y)=\alpha(x, y) \rho(x, y) .
$$

Then $(X, d)$ is a $K$-relaxed polygonal metric space. 
Proof. From the definition of $d$, we have

$$
\rho(x, y) \leq d(x, y) \leq K \rho(x, y)
$$

for $x, y \in X$. By Lemma 5.2, we obtain the desired result.

Example 5.1. Put $X=[0,1)$ and define a function $\rho$ from $X \times X$ into $[0, \infty)$ by

$$
\rho(x, y)=\min \{|x-y|, 1-|x-y|\}
$$

for $x, y \in X$. Let $\theta \in \mathbb{R} \backslash \mathbb{Q}$. Define an equivalence relation $\sim$ on $X$ as in Lemma 5.1. Let $C$ be the quotient mapping from $X$ onto $X / \sim$. Consider $(X / \sim) \subset X$. Define functions $\beta$ and $\gamma$ from $X$ into $\mathbb{Z}$ as follows:

$$
\beta(x)=k, \gamma(x)=\ell \quad \text { iff } \quad x-C x=k \theta+\ell .
$$

Let $\alpha$ be a strictly decreasing function from $\mathbb{Z}$ into $(1,2)$ and define a function $d$ from $X \times X$ into $[0, \infty)$ by

$$
d(x, y)=(\alpha \circ \beta(x)+\alpha \circ \beta(y)) \rho(x, y) .
$$

Define a mapping $T$ on $X$ by

$$
T x=(x+\theta)-[x+\theta] .
$$

Then the following assertions hold:

(i) $(X, \rho)$ is a compact metric space.

(ii) $(X, d)$ is a p-metric space.

(iii) $d(T x, T y)<d(x, y)$ for any $x, y \in X$ with $x \neq y$.

(iv) $T$ does not have a fixed point.

Proof. By Lemma 5.1 (i), $\sim$ is an equivalence relation on $X$. By Lemma 5.1 (ii), $\beta$ and $\gamma$ are well defined.

(i) and (iv) obviously hold. By Lemma 5.3, we obtain (ii).

Let us prove (iii). We have

$$
\begin{aligned}
T x-C x & =x+\theta-C x-[x+\theta] \\
& =(\beta(x)+1) \theta+\gamma(x)-[x+\theta]
\end{aligned}
$$

and hence

$$
\beta(T x)=\beta(x)+1
$$

for $x \in X$. For $x, y \in X$ with $x \neq y$, we have

$$
\begin{aligned}
d(T x, T y) & =(\alpha \circ \beta(T x)+\alpha \circ \beta(T y)) \rho(T x, T y) \\
& =(\alpha(\beta(x)+1)+\alpha(\beta(y)+1)) \rho(x+\theta-[x+\theta], y+\theta-[y+\theta]) \\
& =(\alpha(\beta(x)+1)+\alpha(\beta(y)+1)) \rho(x, y) \\
& <(\alpha \circ \beta(x)+\alpha \circ \beta(y)) \rho(x, y) \\
& =d(x, y) .
\end{aligned}
$$

We have shown (iii). 


\section{OTHER RESUlts}

We finally give an alternative proof of Theorem 4.2 by using Corollary 4.1.

Using Lemma 5 in Jachymski [9], we can easily prove the following proposition.

Proposition 6.1. Let $Q$ be a subset of $[0, \infty)^{2}$. Then the following are equivalent:

(i) $Q$ is Browder.

(ii) For any $r \in(0,1)$, there exists a continuous, strictly increasing function $\eta$ from $[0, \infty)$ into itself satisfying $\eta(0)=0$ and $\eta(u) \leq r \eta(t)$ for all $(t, u) \in Q$.

(iii) There exist $r \in(0,1)$ and a continuous, nondecreasing function $\eta$ from $[0, \infty)$ into itself satisfying $\eta^{-1}(0)=\{0\}$ and $\eta(u) \leq r \eta(t)$ for all $(t, u) \in Q$.

Remark 6.1. In [9], the statement is " $\eta$ is nondecreasing". However, from the proof of Lemmas 3 and 5 in [9], we have that $\eta$ is strictly increasing.

In the remainder of this section, we let $\eta$ be a function from $[0, \infty)$ into itself. We define the following condition:

(H1) For any sequence $\left\{a_{n}\right\}$ in $[0, \infty), \lim _{n} \eta\left(a_{n}\right)=0 \Leftrightarrow \lim _{n} a_{n}=0$. (See Lemma 6 in Jachymski [9])

Lemma 6.1 (Lemma 2.1 in [24]). Let $\eta$ be a continuous, strictly increasing function with $\eta(0)=0$. Then (H1) holds.

Lemma 6.2 (Lemma 2.2 in [24]). Let $\eta$ satisfy (H1). Then $\eta^{-1}(0)=\{0\}$ holds, that is, $\eta(\alpha)=0 \Leftrightarrow$ $\alpha=0$.

Lemma 6.3 ([21]). Let $(X, d)$ be a semimetric space and let $\eta$ satisfy (H1). Define a function $p$ from $X \times X$ into $[0, \infty)$ by $p=\eta \circ d$. Let $\left\{x_{n}\right\}$ be a sequence in $X$ and let $x \in X$. Let $\kappa \in \mathbb{N}$. Then the following hold:

(i) $(X, p)$ is a semimetric space.

(ii) $\left\{x_{n}\right\}$ converges to $x$ in $(X, d)$ iff $\left\{x_{n}\right\}$ converges to $x$ in $(X, p)$.

(iii) $(X, d)$ is sequentially compact iff $(X, p)$ is sequentially compact

(iv) $(X, d)$ is $\kappa$-Hausdorff iff $(X, p)$ is $\kappa$-Hausdorff.

Proof. (i) and (ii) are proved in [21]. Using (H1), we can easily prove (iii) and (iv).

We give an alternative proof of Theorem 4.2 by using Corollary 4.1.

Proof of Theorem 4.2. By Proposition 4.1, $T$ is Browder. Define a subset $Q$ of $[0, \infty)^{2}$ by (3.1). By Proposition 3.2 (iii), $Q$ is Browder. Fix $r \in(0,1)$ and choose $\eta$ appearing in Proposition 6.1 (ii). Define a subset $R$ of $[0, \infty)^{2}$ by

$$
R=\{(\eta \circ d(x, y), \eta \circ d(T x, T y)): x, y \in X\} .
$$

Then we note that $R$ is contractive. Since $\eta$ is continuous and strictly increasing and $\eta(0)=0$ holds, $\eta$ satisfies (H1) by Lemma 6.1. By Proposition 2.1, $(X, d)$ is 2-Hausdorff. Define $p$ by $p=\eta \circ d$. By Lemma 6.3, $(X, p)$ is sequentially compact, 2-Hausdorff semimetric space. By Propositions 3.2 (iv), $T$ is a contraction on $(X, p)$. Therefore we have shown all the assumptions of Corollary 4.1. By Corollary 4.1, we obtain the desired result. 


\section{Acknowledgment}

This paper was supported in part by JSPS KAKENHI Grant Number 16K05207 from Japan Society for the Promotion of Science.

\section{REFERENCES}

[1] S. Banach, Sur les opérations dans les ensembles abstraits et leur application aux équations intégrales, Fund. Math. 3 (1922), 133-181.

[2] F. E. Browder, On the convergence of successive approximations for nonlinear functional equations, Indag. Math. 30 (1968), 27-35.

[3] R. Caccioppoli, Un teorema generale sull'esistenza di elementi uniti in una transformazione funzionale, Rend. Accad. Naz. Lincei 11 (1930), 794-799.

[4] Lj.B. Ćirić, A new fixed-point theorem for contractive mappings, Publ. Inst. Math. (Beograd) 30 (1981), 25-27.

[5] M. Edelstein, On fixed and periodic points under contractive mappings, J. London Math. Soc. 37 (1962), 74-79.

[6] R Fagin, S. R. Kumar, D. Sivakumar, Comparing top $k$ lists, SIAM J. Discrete Math. 17 (2003), 134-160.

[7] M. Hegedüs, T. Szilágyi, Equivalent conditions and a new fixed point theorem in the theory of contractive type mappings, Math. Japon. 25 (1980), 147-157.

[8] J. Jachymski, Equivalent conditions and the Meir-Keeler type theorems, J. Math. Anal. Appl. 194 (1995), 293-303.

[9] J. Jachymski, Remarks on contractive conditions of integral type, Nonlinear Anal. 71 (2009), 1073-1081.

[10] J. Jachymski, J. Matkowski, T. Świątkowski, Nonlinear contractions on semimetric spaces, J. Appl. Anal. 1 (1995), 125134.

[11] M. Kikkawa, T. Suzuki, Fixed point theorems for new nonlinear mappings satisfying Condition (CC), Linear Nonlinear Anal. 1 (2015), 37-52.

[12] W. A. Kirk, N. Shahzad, Fixed point theory in distance spaces, Springer (2014).

[13] W. A. Kirk, N. Shahzad, Fixed points and Cauchy sequences in semimetric spaces, J. Fixed Point Theory Appl. 17 (2015), 541-555.

[14] W. A. Kirk, N. Shahzad, Contractive conditions in semimetric spaces, J. Nonlinear Convex Anal. 17 (2016), $2197-2213$.

[15] J. Matkowski, Fixed point theorems for contractive mappings in metric spaces, Časopis Pěst. Mat. 105 (1980), 341-344.

[16] T. Suzuki, A new type of fixed point theorem in metric spaces, Nonlinear Anal. 71 (2009), 5313-5317.

[17] T. Suzuki, Discussion of several contractions by Jachymski's approach, Fixed Point Theory Appl. 2016 (2016), Article ID 91.

[18] T. Suzuki, Another generalization of Edelstein's fixed point theorem in generalized metric spaces, Linear Nonlinear Anal. 2 (2016), 271-279.

[19] T. Suzuki, Characterizations of contractive conditions by using convergent sequences, Fixed Point Theory Appl. 2017 (2017), Article ID 30.

[20] T. Suzuki, Generalizations of Edelstein's fixed point theorem in compact metric spaces, Fixed Point Theory, in press.

[21] T. Suzuki, Fixed point theorems for contractions in semicomplete semimetric spaces, submitted.

[22] T. Suzuki, Characterization of $\sum$-semicompleteness via Caristi's fixed point theorem in semimetric spaces, J. Funct. Spaces 2018 (2018), Article ID 9435470p.

[23] T. Suzuki, B. Alamri, M. Kikkawa, Edelstein's fixed point theorem in generalized metric spaces, J. Nonlinear Convex Anal. 16 (2015), 2301-2309.

[24] T. Suzuki, M. Kikkawa, Generalizations of both Ćirić's and Bogin's fixed point theorems, J. Nonlinear Convex Anal. 17 (2016), 2183-2196.

[25] T. Suzuki, M. Kikkawa, C. Vetro, The existence of best proximity points in metric spaces with the property UC, Nonlinear Anal. 71 (2009), 2918-2926. 